\title{
Fast-Response Infrared Ferroelectric Liquid Crystal Phase Modulators
}

\author{
Ju-Hyun Lee \\ Yung-Hsun Wu \\ Shin-Tson Wu
}

College of Optics and Photonics, University of Central Florida,

Orlando, Florida, USA

\section{Dong-Woo Kim \\ Chang-Jae Yu \\ Sin-Doo Lee}

School of Electrical Engineering, Seoul National University, Kwanak, Seoul, Korea

\begin{abstract}
A high speed phase modulator based on ferroelectric liquid crystal (FLC) is demonstrated. For uniform alignment and pure phase modulation, we propose a new FLC device using a short helical pitch material in a homeotropic alignment. This device is driven by periodic in-plane electrode stripes implemented on the surface of both cell substrates. As a result, we have obtained a large phase modulation (about $2.3 \pi$ at $\lambda=1.55 \mu \mathrm{m}$ under $E=2.5 \mathrm{~V} / \mu \mathrm{m})$ and fast response time $(<200 \mu \mathrm{sec})$. Together with the performance characterization of our device, we discuss the alignment breaking effect which occurs near the edges of operating region.
\end{abstract}

Keywords: alignment breaking effect; ferroelectric liquid crystal; IR application; phase modulation; vertical alignment

High-speed phase modulation using liquid crystals (LCs) is desirable for many photonic applications [1,2]. Among various LC phases, nematic is widely used for displays, light shutters, and optical communications [3]. The major advantages of nematics are analog phase modulation capability and easiness of getting uniform molecular alignment. However, nematic is known to have slow response time because

Address correspondence to Shin-Tson Wu, College of Optics and Photonics, University of Central Florida, Orlando, FL 32816, USA. E-mail: swu@mail.ucf.edu 
of the collective molecular reorientation. In addition, this slow response gets worse if the LC layer thickness increases in order to obtain a $2 \pi$ phase change at an infrared wavelength, say $\lambda=1.55 \mu \mathrm{m}$.

On the contrary, ferroelectric liquid crystals (FLCs) show very fast response time because their switching dynamics is different from that of nematic liquid crystals. However, most of the FLC modes are not suitable for pure phase modulation devices because their optic axis sweeps in the plane parallel to the cell substrate so that it changes the polarization state of the incident light. Moreover, many FLC modes exhibit bistable switching [4] that cannot be applied to the analog phase modulator. The most serious problem in FLC devices is that obtaining the alignment uniformity with optical quality is almost unattainable.

In this paper, we demonstrate a new FLC device configuration which shows a large phase modulation, fast response, and good alignment uniformity. Together with results of the device performance characterization, we discuss an alignment breaking phenomenon which appears near the edges of the operation region.

Figure 1 depicts the device structure employed in this study. We adopt the vertically aligned short pitch FLC configuration that was proposed as a transverse electro-optic effect in $\mathrm{SmC}^{*}$ material in late 80s [5] and was re-examined for its potential display applications

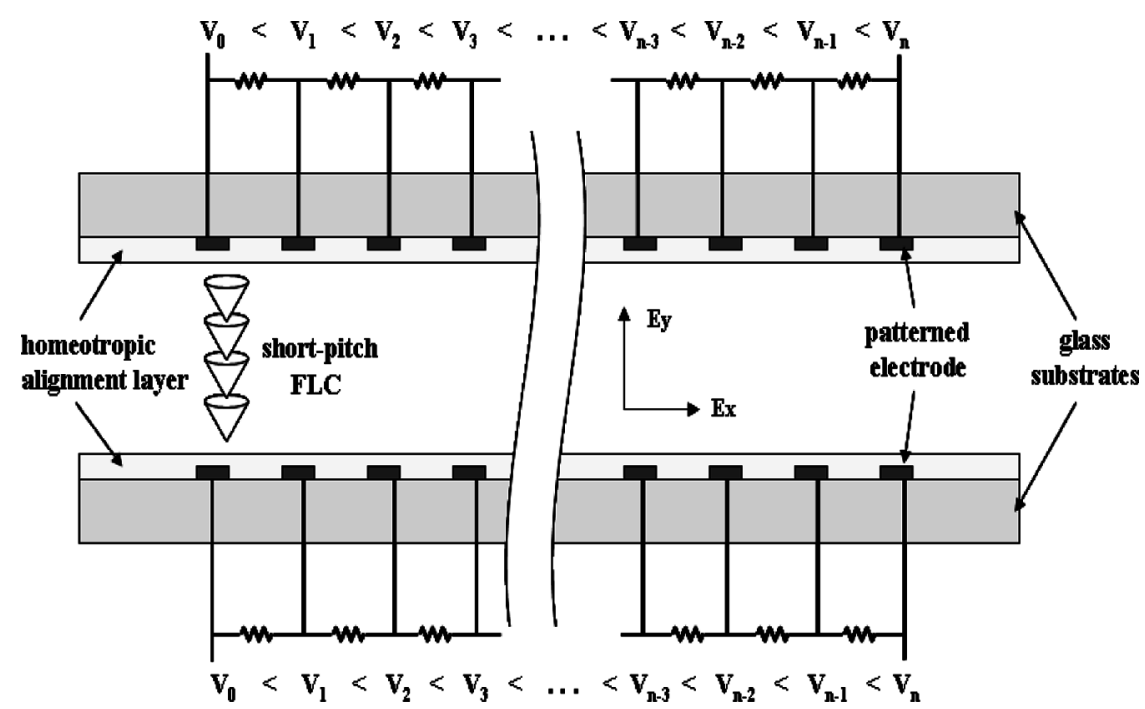

FIGURE 1 Device structure for the fast phase modulator application using vertically aligned short pitch FLC configuration. 
due to its very uniform molecular alignment and wide viewing angle originated from in-plane electrodes [6]. However, for the device driven by conventional in-plane electrodes, every other optic axis of the active region alternates because the voltage applied to the electrode stripes alternates. It induces fringe field near the edge of every electrode stripe. Moreover, if we use the single-side electrode structure, the transverse electric field strength decreases as it moves away from the electrode implemented side. This situation forms a phase grating in the whole operating region and limits the ability to obtain large phase retardation. To solve these problems, we implement the periodic in-plane electrode stripes on the inner surfaces of the top and the bottom substrates as shown in Figure 1. The electrode stripes are electrically connected by thin film resistor network. Because of the resistor network, the monotonous increasing/decreasing voltages are applied across the electrodes. In addition, the electrode patterns on both substrates are aligned precisely to hold the same voltage on the electrode stripes placed opposite to the substrates. Therefore, the equal-potential lines would align normally to the cell substrates and the uniform transverse electric field $\left(\mathrm{E}_{\mathrm{x}}\right)$ without vertical component $\left(\mathrm{E}_{\mathrm{y}}\right)$ can be obtained in certain range of the operating region. As a result, uniform phase retardation can be obtained in the operating region.

Based on this device configuration, we fabricated a cell with short pitch FLC material, FLC 10817, purchased from Rolic Technologies. Its phase transition has following sequence: isotropic $\rightarrow\left(64.5 \sim 62.4^{\circ} \mathrm{C}\right)$ $\rightarrow$ cholesteric $\rightarrow\left(62.4 \sim 61.5^{\circ} \mathrm{C}\right) \rightarrow$ smectic $\mathrm{C}^{*}$. At room temperature, the spontaneous polarization, the smectic tilt angle, and the helical pitch of FLC-10817 are $115 \mathrm{nC} / \mathrm{cm}^{2}, 34^{\circ}$, and less than $0.2 \mu \mathrm{m}$, respectively. The sample cell was made with glass substrates coated with patterned indium-tin-oxide electrodes. Polyimide JALS 204 (Japan Synthetic Rubber Co.) was coated on the substrate surfaces to promote homeotropic alignment. Neither surface was rubbed.

Comparing with the nematic vertical alignment (VA) mode, vertically aligned FLC mode has a limit in the maximum switching angle of the optic axis. In a nematic LC cell, the LC directors can be completely reoriented. However, the average optic axis in our FLC sample switches narrower than the smectic tilt angle. Therefore, the effective birefringence for the normal incident light is reduced. To compensate for this effect and to obtain a large phase change, a thick LC layer is required. We have prepared a cell with $50 \mu \mathrm{m}$ gap. The electrode width and gap are both controlled at $5 \mu \mathrm{m}$. The driving voltage is applied to the zeroth and the tenth electrode stripes, so that the total width of operating region is $100 \mu \mathrm{m}$. 


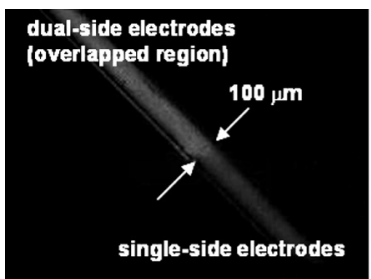

(a)

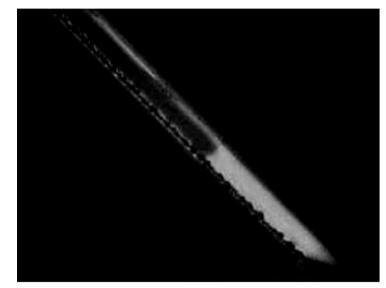

(d)

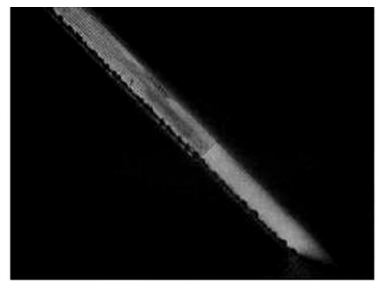

(b)

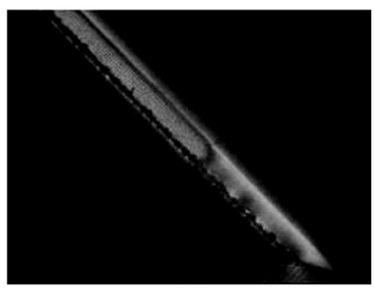

(e)

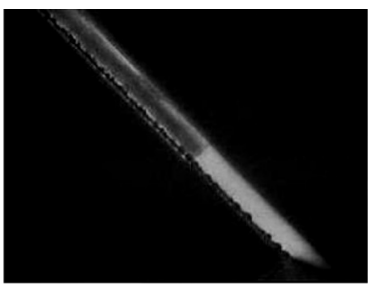

(c)

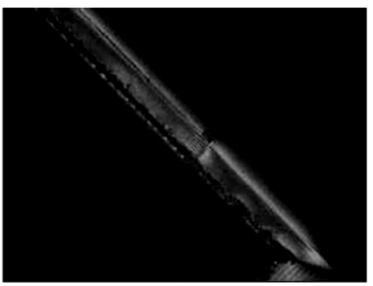

(f)

FIGURE 2 Alignment texture variation on the operation region according to the applied voltage change: (a) $\mathrm{V}_{\mathrm{p}}=90$, (b) 110 , (c) 120 , (d) 130 , (e) 150, and (f) 170 volts, respectively.

Figure 2 shows the LC texture photographs taken by a polarizing optical microscope under different driving voltages. The upper-left part of the operating region has the electrode patterns on both substrates. The lower-right part has single side electrode pattern. The electro-optic property was measured in the upper-left part. Textures were observed under crossed polarizers. The angle between the optic axis of the polarizer and the electrode stripes is 45 degrees. Bi-polar square waveform of $60 \mathrm{~Hz}$ was applied to operate the sample. In the figure, the non-operated region shows a very good dark state, indicating that the vertically aligned short pitch FLC structure looks like a vertical aligned nematic LC in the visible spectral region. This is because the visible wavelength is longer than the helical pitch length of the FLC so that the incident light cannot resolve the helical structure and only sees the average optic axis of the FLC. In the operating region, light transmittance oscillates with the applied voltage. The first maximum occurs at $\sim 110 \mathrm{~V}$. Above $110 \mathrm{~V}$, the transmittance decreases gradually and reaches the darkest state at $\sim 130 \mathrm{~V}$. Above $130 \mathrm{~V}$, the transmittance increases, but reaches another dark state at $170 \mathrm{~V}$. This result indicates that the phase retardation of the FLC cell is $\sim 2 \pi$ at 130 volts and $4 \pi$ at $V \sim 170$ volts at the employed green light. One interesting phenomenon observed is the broken alignment near the edge of the operating region. 
To measure the electro-optical performance of the device at infrared, we used a diode laser with $\lambda=1.55 \mu \mathrm{m}$ as a probe beam. To avoid the effect of alignment breaking on the measurement, we used a convex lens to focus the laser beam to the center of the operating region. Figure 3(a)

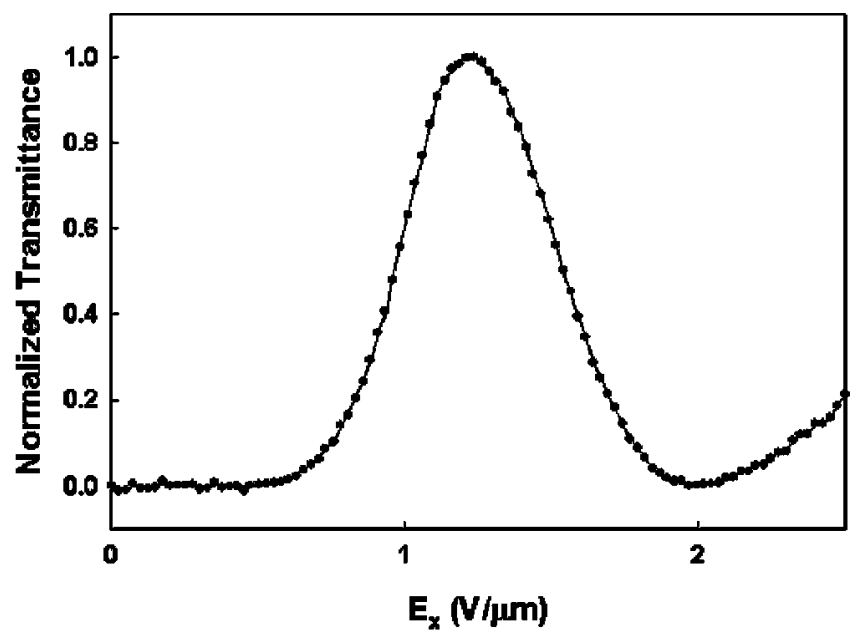

(a)

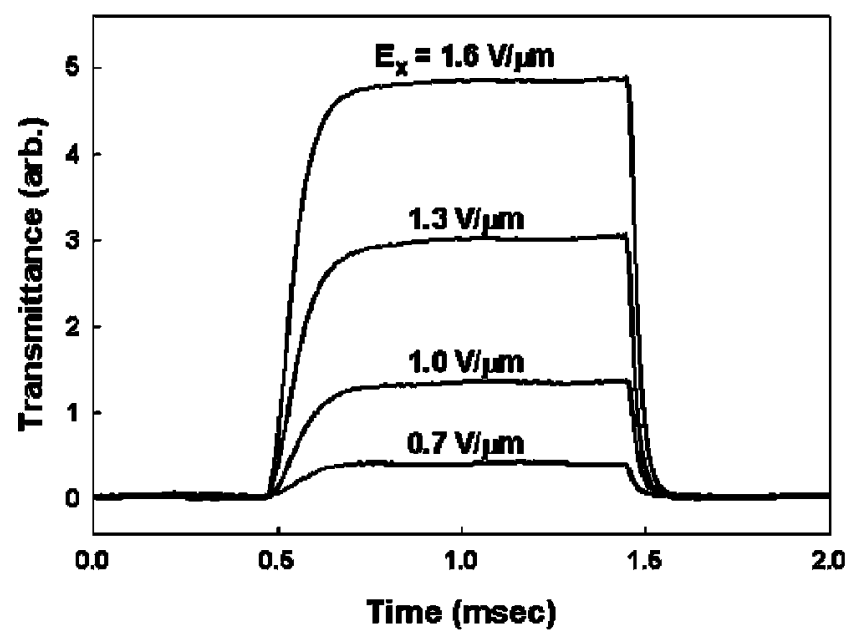

(b)

FIGURE 3 Electro-optic characteristics of the proposed device: (a) voltage dependent transmittance variation, and (b) dynamic response of the transmittance for unipolar square-wave voltage bursts. 
shows the normalized V-T curve of our device. The maximum phase retardation was calculated from the normalized transmittance $T=\sin ^{2}(\delta / 2)$. The obtained result is $\delta=2.3 \pi$ at $\lambda=1.55 \mu \mathrm{m}$ and $\mathrm{E}_{\mathrm{x}}=2.5 \mathrm{~V} / \mu \mathrm{m}$. Above $\mathrm{E}_{\mathrm{x}}=2.5 \mathrm{~V} / \mu \mathrm{m}$, the alignment breaking worsens and permeates into the measurement region so that the V-T curve fluctuates severely. We also measured the response time of our device. To measure the turn-on (rise) and the turn-off (decay) time, we applied unipolar square voltages. Figure 3(b) shows that the rise and decay time is $\sim 110 \mu \mathrm{s}$ and $\sim 60 \mu \mathrm{s}$, respectively. We also measured the response time of the $18-\mu \mathrm{m}$-thick sample and the results are $\sim 80 \mu \mathrm{s}$ rise and $60 \mu$ s decay. From these results, we conclude that the response time of our device is insensitive to the cell gap and the electric field strength. This result is quite different from that of conventional FLC devices. Our helix-deformation-based vertical aligned FLC is supposedly free from the surface effect which affects the electro-optic response of the thin and planar FLC because it is driven by the lateral electric field and the FLC layer is relatively thick.

Figure 4 shows the texture of alignment breaking in detail after the device was driven by a strong electric field, $\mathrm{E}_{\mathrm{x}}>2 \mathrm{~V} / \mu \mathrm{m}$. Alignment breaking on the left side area $\mathrm{A}$ is more severe than that on the right side area $\mathrm{B}$. The shape of broken alignment is similar to the beads in a line.

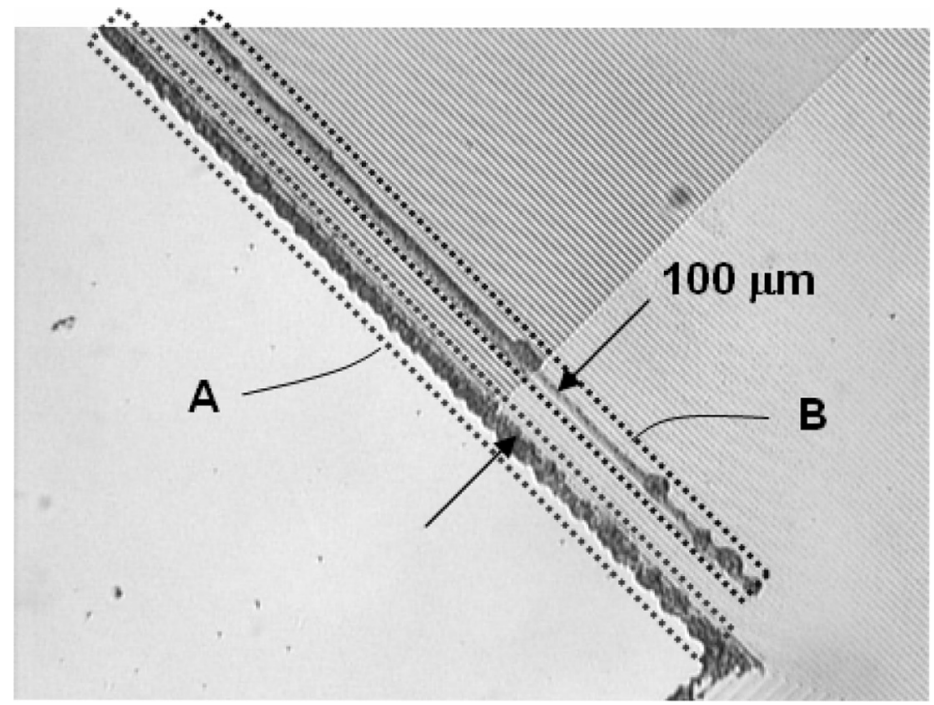

FIGURE 4 Texture of broken alignment after the device has been driven by a strong electric field. 
To understand the broken alignment phenomenon, we simulated the electric field and the electric potential distribution. The upper graph of Figure 5 shows the vertical component of the electric field
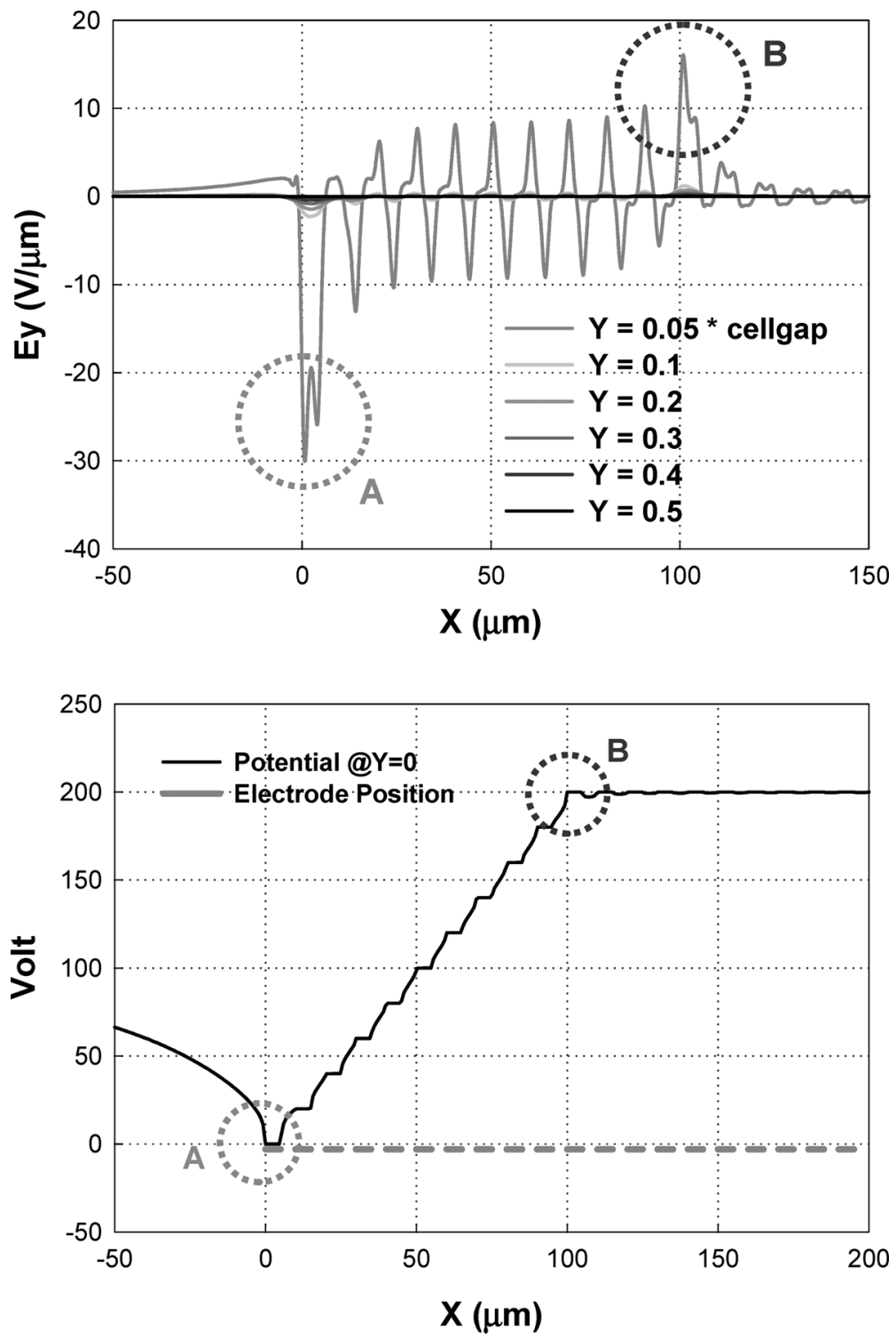

FIGURE 5 Simulation results of $\mathrm{E}_{\mathrm{y}}$ at different y positions and the electric potential in the vicinity of the substrate surface. 
$\left(\mathrm{E}_{\mathrm{y}}\right)$ across the operating region at different distances from the substrate. As shown in the figure, the fringe field remains very strong near the surface region. In addition, the $\mathrm{E}_{\mathrm{y}}$ in area $\mathrm{A}$ is much stronger than that in area $\mathrm{B}$. The $\mathrm{E}_{\mathrm{y}}$ in area $\mathrm{B}$ is also stronger than that in other operating region. From this result, we conclude that the vertical component of the electric field, $\mathrm{E}_{\mathrm{y}}$, induces the alignment breaking and it has a certain threshold value to make the alignment breaking with a shape of beads-line. The reason why the $\mathrm{E}_{\mathrm{y}}$ in $\mathrm{A}$ is stronger than that in $\mathrm{B}$ is because the electric potential in A varies abruptly but that in $\mathrm{B}$ does not due to the resistance network connecting with other electrode stripes.

Figure 6 shows a physical model to explain the alignment breaking phenomenon which appears in the edges of the operating region.

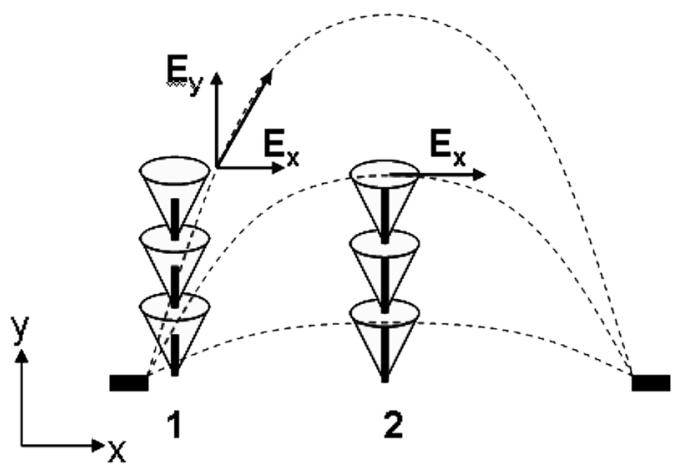

(a)

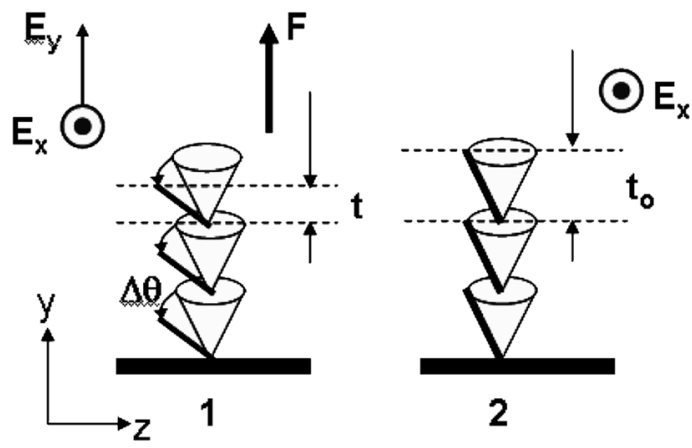

(b)

FIGURE 6 Model for explaining the localized layer buckling phenomenon: (a) front view and (b) side view of the operation region. 
Figure 6(a) is the front view of the device. Black rectangles are the cross-section of two in-plane electrode stripes. The applied voltage generates fringe field. Near the center (position 2) between two electrode stripes, only the lateral electric field component exists. However, near the edges (position 1) both vertical and lateral components of the electric fields exist. Because the FLC molecules exhibit a spontaneous polarization whose direction is normal to the plane formed by the helix axis and the long axis of the molecule, switching direction of the FLC molecules is on the $\mathrm{x}-\mathrm{z}$ plane, as shown in the figure. Figure 6(b) shows the side view of above situation. In position 2 , all FLC molecules are switched by a strong lateral electric field in the same direction. The maximum switching angle in this position is same to the smectic tilt angle because of the interaction between the molecular dipoles and the lateral field $\mathrm{E}_{\mathrm{x}}$. And the layer thickness is remained to the original smectic layer thickness $t_{0}$. On the contrary, in position 1 the interaction between the negative dielectric anisotropy of the molecule and the vertical electric field $\mathrm{E}_{\mathrm{y}}$ exists. We confirmed by dielectric measurements using parallel and vertical aligned cells that indeed the employed FLC has a large negative dielectric anisotropy, $\Delta \varepsilon \sim-20$. Therefore, the molecules are inclined further and this additional inclination $\Delta \theta$ reduces the equilibrium layer thickness from $t_{0}$ to $t$. However, the material tends to keep its original layer thickness and this tendency induces a dilative strain [7-9] along the y direction. This dilative strain leads to a buckling of the layers.

To confirm the dielectric-coupling-based molecular inclination along the edges in the operating region, we made a cell with single side inplane electrodes and a thin cell gap. Using this sample, we observed the variation of the texture and the transmittance distribution as a function of applied voltage as shown in Figure 7. In Figure 7, the dark regions are covered by transparent electrodes and there is no electric

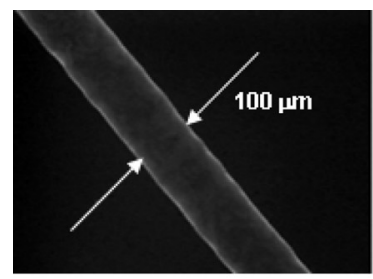

(a)

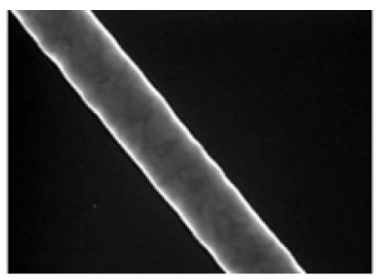

(b)

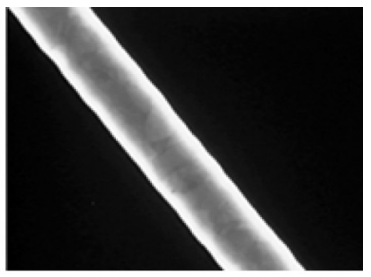

(c)

FIGURE 7 Microscope photos of textures on the operating region of the vertically-aligned FLC cell with single sided in-plane electrodes. The applied voltages are (a) $80 \mathrm{~V}$, (b) $120 \mathrm{~V}$, and (c) $200 \mathrm{~V}$. 
field over there. Therefore, no molecular switching is induced in this area so that no transmittance is detected under crossed polarizers; only the electric field is induced between two electrodes. The gap between two electrodes is $100 \mu \mathrm{m}$ and the cell gap is $5 \mu \mathrm{m}$. Because the electrodes are only on one substrate, the phase retardation is lower than that of the cell which has electrodes on both substrates [10]. Consequently, the experimental condition leads to a low transmittance at the center of the operating region. On the contrary, high transmittance was observed in the area close to the electrode edges. Considering the thin cell gap, weak electric field strength, and the limit of the switching angle of the average optic axis confined by the smectic tilt angle, the bright state transmittance is anomalous. This anomalous behavior is explained by the switching of the optic axis larger than smectic tilt angle due to a large dielectric coupling energy.

The molecular switching in which the angle is larger than the smectic tilt angle induces the dilative strain and the layer buckling phenomenon as mentioned above. For the case of uniform dilative strain, it was well known that the displacement field with respect to buckling has been analyzed previously [9] as follows: $u(x, y) \propto$ $\cos \left(q_{y} y\right) \cos \left(q_{x} x\right)$, where $q_{y}=\pi / L_{y}, q_{x}=2 \pi / \Lambda, L_{y}$ is a cell gap, and $\Lambda$ is a buckling period.

However, in our sample the electric field distribution is localized on the surface and the electrode edge region so that the dilative strain is not uniform. Using a crude assumption that the additional inclination of the switching angle is a function of the electric field strength above certain threshold value, we could modify the amplitude of the displacement formula in the surface region within a thin thickness so that we can ignore the $\mathrm{E}_{\mathrm{y}}$ variation along $\mathrm{y}$ direction:

$$
u(x, y, z) \propto \cos \left(q_{y} y\right) \cos \left(q_{z} z\right) f\left(\left|E_{y}(x)-E_{t h}\right|\right)
$$

Using this equation together with the simulated electric field distribution results, we estimate the localized buckling shape qualitatively as Figure 8(a) shows. Figure 8(b) plots the two dimensional mapping of the displacement field of the layer surface A. If the electric field strength increases so that this localized buckling is intensified further, continuum of the smectic layer between the buckled region and unbuckled region would not be sustained any longer. As a result, the layer breaking would occur. If the layer breaking is induced along a contour of the displacement field, the broken texture would be shaped as a beads-line as we observed in Figure 4.

In conclusion, we have demonstrated a new FLC device structure based on the vertically aligned short-pitch FLC mode. Employing 


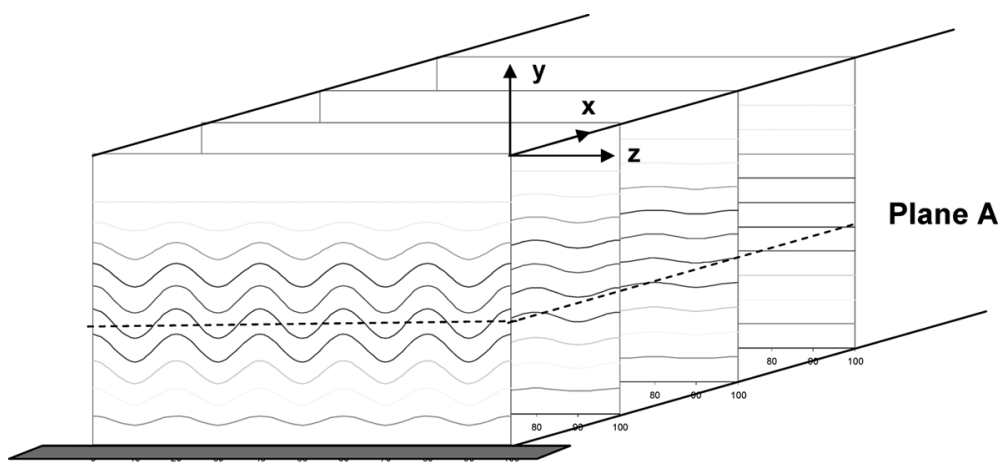

(a)

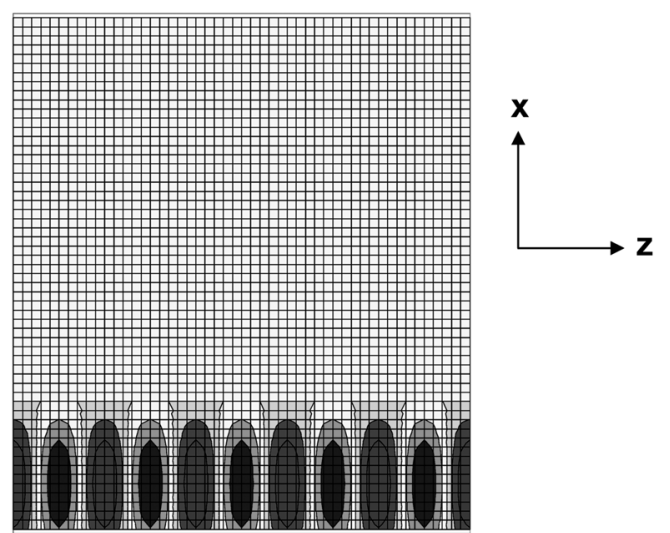

(b)

FIGURE 8 Simulation results of (a) localized smectic layer buckling phenomenon, and (b) two dimensional mapping of the deviation of the layer surface A.

the in-plane electrode stripes which are implemented on the surface of both top and bottom substrates and electrically connected by the thin film resistor network, fast response, large phase modulation with continuous gray scales have been obtained. In addition, good alignment uniformity is achieved by a simple fabrication process. It thus would be useful for photonic device applications such as a fast response spatial phase modulator. In addition, we have studied the alignment breaking effect which appears in the edges of the operating region. The alignment breaking is explained by the excessive layer buckling due to the dielectric coupling between the negative dielectric anisotropy of the employed material and the vertical component of the electric field. 


\section{REFERENCES}

[1] Wu, Y. H., Lin, Y.-H., Ren, H., Nie, X., Lee, J. H., \& Wu, S. T. (2005). Opt. Express, $13,4638$.

[2] Ren, H., Lin, Y. H., Fan, Y. H., \& Wu, S. T. (2005). Appl. Phys. Lett., 86, 141110.

[3] Wu, S. T. \& Yang, D. K. (2001). Reflective Liquid Crystal Displays, Wiley: New York.

[4] Clark, N. A. \& Lagerwall, S. T. (1980). Appl. Phys. Lett., 36, 899.

[5] Chigrinov, V. G., Denisov, A. F., Parfenov, A. V., \& Pozhidaev, E. P. (1988). Ferroelectrics, 85, 303.

[6] Lee, J. H., Yu, C. J., You, D. H., \& Lee, S. D. (1999). Proc. Int'l Display Workshop, 129.

[7] Singer, S. J. (1993). Phys. Rev. E, 48, 2796.

[8] Geer, R. E., Singer, S. J., Selinger, J. V., Ratna, B. R., \& Shashidhar, R. (1998). Phys. Rev. E, 57, 3059.

[9] Clark, N. A. \& Meyer, R. B. (1973). Appl. Phys. Lett., 22, 493.

[10] You, D. H., Lee, S. D., Lee, J. H., \& Na, D. J. (2001). J. SID, $2,1$. 